\title{
An Improved Local Binary Patterns Histograms Technique for Face Recognition for Real Time Applications
}

\author{
Akshit Anand, Vikrant Jha, Lavanya Sharma
}

\begin{abstract}
Recently, face recognition and its applications has been considered as one of the image analysis most successful applications, especially over the past several years. Face Recognition is a unique system that can be used by using unique facial features for identification or verification of a person from a digital image. In a face recognition system, there are many technique that can be used. This paper provides an efficient of the Local Binary Patterns Histograms (LBPH) based technique provided by OpenCV library which is implemented in Python programming language which is well suitable for realistic scenarios. In this paper we also provide visual qualitative outcome with existing algorithm (Haar-cascade classifier and Local Binary Patterns Histograms (LBPH)). As a result, the proposed technique outperform better in terms of visual qualitative analysis.
\end{abstract}

Index Terms: Face Recognition, Face Detection, Local Binary Patterns Histograms, OpenCV, Haar-Cascade Classifier, Python.

\section{INTRODUCTION}

The information age is rapidly revolutionizing the completion of transactions. In our day-to-day life security is our major concern because everyday actions are being handled increasingly electronically, rather than face - to - face with pencil and paper. This increase in electronic transactions resulted in increased demand for quick and accurate identification and authentication of users. Access codes often use PINs for identification and security clearances for buildings, bank accounts and computer systems. Using the correct PIN will gain access, but it will not verify the PIN user. Biometrics systems provide assistance in solving this issue. In biometric software applications face recognition is a well - greeted and reviewed with approval application that can identify verified faces \& verify reviews from a given database place among the various biometrics available. Face recognition recognize faces through frame from the video or images. Face recognition has used for verification or identification a person in security and commercial systems and is better than others biometric systems such as retinal or iris scans and analysis of fingerprints. A well - known

Revised Manuscript Received on July 5, 2019

Akshit Anand, Amity Institute of Information Technology/ Amity University/ Noida, India,

Vikrant Jha, Amity Institute of Information Technology/ Amity University/ Noida, India.

Lavanya Sharma, Amity Institute of Information Technology/ Amity University/ Noida, India. technique is used in literature called Local Binary Patterns Histograms. Which is used to extract and implement face features that are then proceed and compared in the database, if the face reviews exists or the similarity of the face is under database then the system will be able to show the image or that will be not so known or not available in the database $[1,4,5]$. Local Binary Pattern is an easy step but well-organized texture operator that tags a picture element by thresholding each pictures neighborhood along with views the outcome as a binary figure. LBP is combined with gradient - oriented histograms (HOG) descriptors, significantly improving detection performance on some datasets. Using the LBP in combination with histograms we can use a simple data vector to represent the face images. Hundreds of images require LBP training. Each image of training is split into a few blocks. It's a visual descriptor as well. LBP characteristics are taken out to form a feature vector that categories a face from a non - face. There are some approaches to increasing the rate of facial recognition. According to the paper we conducts a qualified study of the Local Binary Patterns Histograms algorithm with the Haar - cascade classifier algorithm using both the algorithm that will be implemented in the programming language of Python to improve face recognition.

\section{RELATED WORK}

Different work on face detection and recognition has been done in literature but there is still a problem so it is a very challenging job to handle face in details for better recognition. L. Zhichao and M. Joo [1] in their work, refered to various current engagements to represent all variation of faces in full detail, also setting side by side all of these engagements, a comprehensive study is carried out on the Local Binary Patterns (LBP) and to examine how face recognition operates under different radiance, as well as different combinations with other techniques. It was observed that when there is only one picture per person, then majority of the system do not provide competent presentation as compared to when it is done with more than one picture per person, so when Local Binary Patterns (LBP) is merged with different techniques, it also makes it easier to deal with variation in illumination. 
M. Abuzneid [2] presented an intensified approach to enhanced human face recognition by making use of Back Propogation Neural Network (BPNN) and extracte attribute build on the interconnection between the training photos. Using a new set from the original training dataset called the T - Dataset that is used to train the BPNN. T Dataset using the training image correlation without using a common picture mass technique. The correspond $\mathrm{T}$ Dataset produce an elevated layer of distinguishing between the training pictures, helping the BPNN to coincide more quickly and attain finer accuracy. Reduction of statistics and attributes are necessary in the face recognition operation and the modern Neural Network (NN) has recently been focused by researchers. Therefore, using a descriptor of the Local Binary Pattern Histogram (LBPH) to demonstrate that even using traditional methods there is potential for improvement.

A Complexion Face texture attributes are obtained through cascading into two Local Binary Pattern features in proposed application by C. Sun and J.,Quanbinli [6]. Then, the use of the SVM classifier achieves face recognition under the light of complex. First, by using relatively small LBP operators, that local particular texture attribute of the sub - block face attributes are quite secured. Then, after using comparison large LBP operators, the global profile quality are accessed. Ultimately, as a final identification feature, two histograms acquired are combined into single correct order.

S. Y.Raut [7] suggested an algorithm based on E - HMM and discerning set, their technique is splitted into 2 sections, firstly, the training module and secondly an assembly module $\&$ the paper has a very high recognition rate set side by side to other techniques.

\section{EXISTING MODEL}

The oldest and most basic model for face detection in OpenCV is the Haar - Classifier model. To perceive multiple facial attributes, the Haar-cascade uses the AdaBoost algorithm. Firstly, it reads the pictures to be noticed and transforms it is in the gray-scale image, then loads the hair cascade categories to determine if it carry a human face. In this case, the facial features will be examined and a rectangular frame will be drawn on the detected face. Otherwise, the next picture is still being tested. It begins by extracting hair characteristics through each image as shown in to following steps.

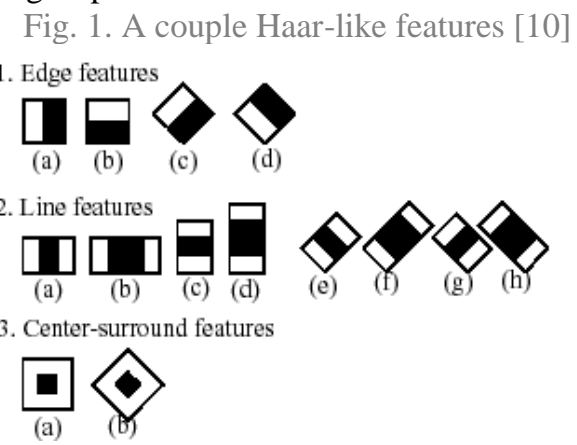

To calculate a single feature, each window is placed on the image. This characteristic or attribute is a single value acquire by subtracting the pixel combination from the sum of the images below the black portion of the window below a white part of that window. Now, to calculate many characteristics, all practicable sizes of every window are set at all possible place or situation of each picture.

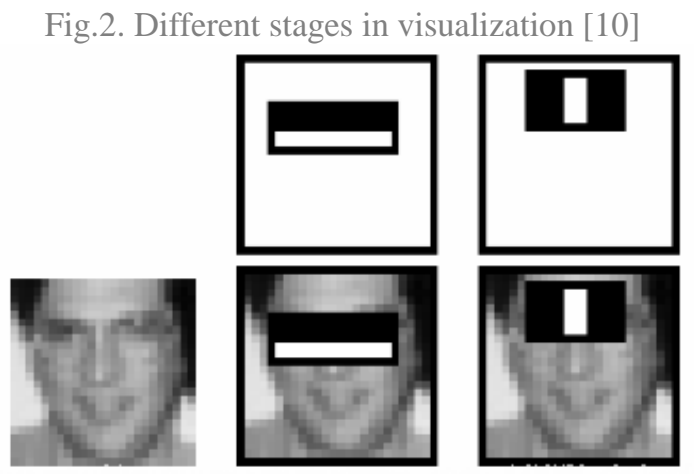

For instance, two features are extracted in the above image. The first one focuses on the thing that the eye region is frequently darker than the nose as well as cheeks on area. The second attribute is that eyes are darker than the nose viaduct, depending on that property. But most of them are irrelevant among all these calculated features. For example, the windows become irrelevant when used on the cheek because no part of these areas on the cheeks are quite darker or lighter than other regions, all sectors are similar. This technique of face detection is the base technique of OpenCV but it has some of its limitation which are listed below -

- Computationally compound and slow

- Longer Training Time

- Low accuracy on black faces

- Limitations on difficult lightning shape

- Low robust to occlusion

The main limitation of this existing technique is it is only used for face detection that mean it is great for detecting faces but not for recognizing the faces for which we are using Local Binary Patterns Histograms (LBPH) technique to store face images in dataset and compare it with the represented face or images.

\section{PROPOSED WORK}

The proposed method is improved the haar-classifier technique by removing its some limitation by combining with the local binary patterns classifier. Using both the technique we can enhance the face recognition. Each picture of face in the face dataset has the individual number of IDs in this experiment. The proposed framework with major steps involved are shown in Fig. The major steps of proposed technique are:

1. Detection of face.

2. Training stage of detected faces.

3. Recognition of detected images. 
Fig.3. Flow Chart of Face Recognition application

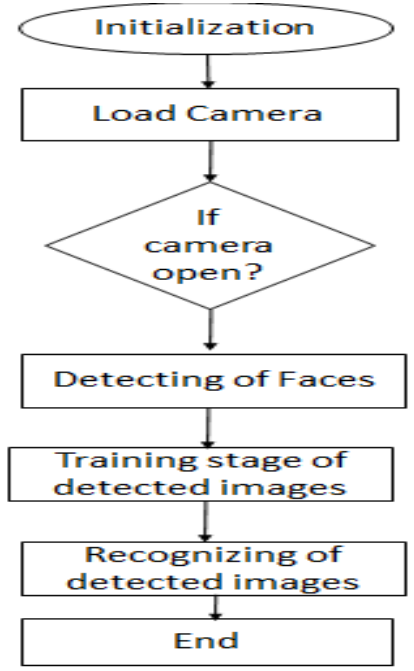

Firstly, ready the dataset face and then take out each test image's LBP texture features. Finally, the facial information is classified and recognized. These face pictures are captured with 720p camera for this test we stored first 21 pictures of a single face. We contrast the input face pictures with the dataset face pictures and check as if the given appeared pictures were not recognized or else the face picture would not be recognized after take out attributes compared to the dataset so that we can finally figure out the face picture. It can be shown in Fig. 4.

Fig. 4. Detection of Unknown Face.

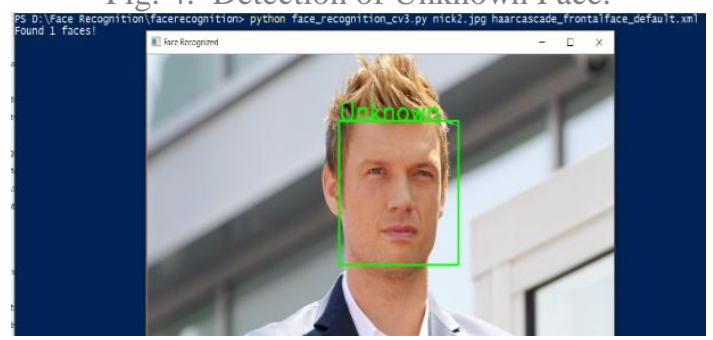

According to the proposed technique, this face picture is compared with the face picture of a familiar face from the available dataset.

1. Detecting of Faces: The structure notices the face through a camera in detailed image and captures the image in black and white filter as shown in Fig. 8

Fig. 5. Detecting of Faces.

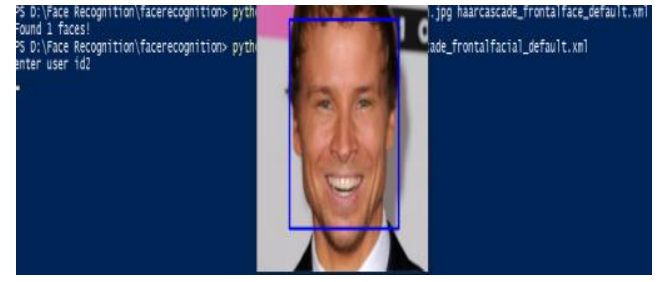

2. Training stage of detected faces : In this step, faces are detected from the datasets training after picture accession and pre - processing task. In this step, the recognizer trainer is used to store the histogram values of faces for the training phase as show in Fig6.

Fig.6. Training stage of detected faces

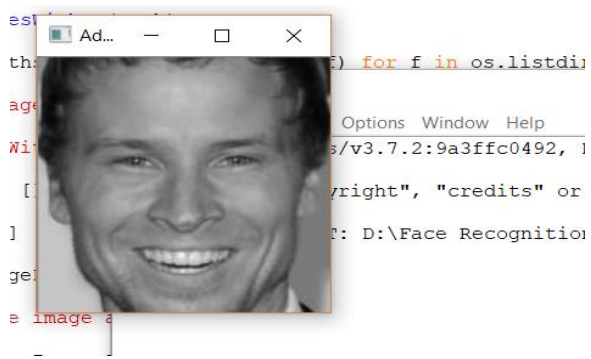

3. Recognition of detected images: Recognizing face images is the final task. The Haar-Cascad and Histogram Training Recognizer for Local Binary Patterns will be used to recognize face. The dataset compares the stored images of the face with the images of the input face. The recognized outcome will be shown on the systems screen to check if the face features of input pictures match the database pictures or not as shown in Fig 7.

Fig. 7. Recognition of detected image

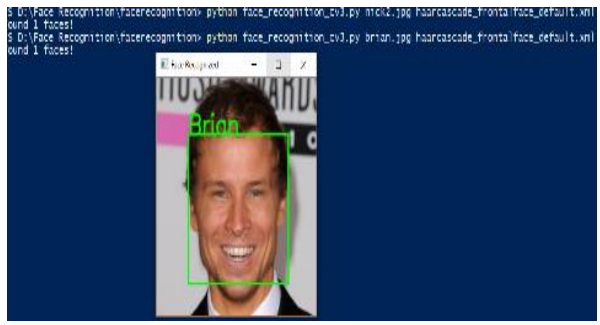


Fig.8. Qualitative Analysis: (a) Dataset and frames number, (b) Original Images, (c) Grayscale Images, (d) Existing Model Results, (e) Proposed Results

\begin{tabular}{|c|c|c|c|c|}
\hline Dataset & $\begin{array}{l}\text { Original } \\
\text { Images }\end{array}$ & $\begin{array}{l}\text { Grayscale } \\
\text { images }\end{array}$ & $\begin{array}{l}\text { Haar- Cascade } \\
\text { technique }\end{array}$ & $\begin{array}{l}\text { Proposed } \\
\text { results }\end{array}$ \\
\hline Frame 1 & 7 & & 717 & $m$ \\
\hline Frame 2. & $110 y$ & & IIU & \\
\hline Frame 3. & & & & \\
\hline Frame 4. & & & & \\
\hline Frame 5. & & & & \\
\hline Frame 6. & & & & \\
\hline Frame 7. & & & & \\
\hline Frame 8 & & E & on & Fans \\
\hline Frame 9. & & & & \\
\hline
\end{tabular}

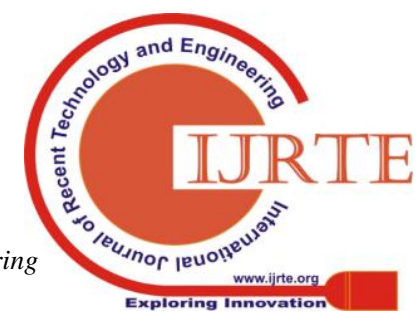


The proposed method leads to better results in visual qualitative. The conventional face detection technique are computationally complex and slow as well as they have high detection accuracy but longer training time, they are less accurate on black faces, limitations in difficult lightening conditions so to remove these limitations we used a better face recognition approach with the traditional face detection approach for better results. As shown in Table1 in the existing model it will only be able to detect faces not recognizing and in the proposed results will not only be able to detect faces but it will also recognize the faces using unique ID's and the grayscale images will be used to train the captured images. All the images will be converted to grayscale images during acquisition for feature extraction.

\section{OBSERVATION}

The proposed technique namely "An Improved Local Binary Patterns Histograms technique for face recognition" this technique has better results in terms of quality as compared to the existing one. The following observations were made on the basis of qualitative analysis as shown in Fig.8:

- Nine no. frames were used in qualitative analysis which can be shown in Table I, where the results proposed are better than the existing model. To achieve better face detection, the unwanted detection of faces was reduced (shown in Fig.10).

- Existing model technique can only detect faces where the proposed technique is capable of not only detecting but also recognizing faces and reducing undesirable detection.

- Set side by side the existing model approach and the proposed approach, the overall accuracy of the proposed approach is maximum.

The overall observation predicts that our technique performs better as compared to the existing model considered

\section{CONCLUSION AND FUTURE WORK}

For face recognition, we used Local Binary Patterns. It contains three main parts, i.e. facial representation, extraction of features, and finally classification. While the input of face behaves is described in Face representation and, moreover, it limits the detection and recognition algorithms. Furthermore, this LBP histogram has produced a new result for feature extraction and finally we can categorize detected face input as compared to the DATASET proposed. Then we can examine either a known person or an unknown person recognized by our system. In the coming future, this proposed model will be more advantageous in identifying criminals with criminal records in the database for security agencies. And this technique help in recognizing any related or unrelated person in the monitoring area at low resolution due to long distance monitoring or security camera and observer subject.

\section{REFERENCES}

1. A. Ahmed, J. Guo, F. Ali, F. deeba. LBPH Based Improved Face recognition At Low Resolution,2018 Internation Conference on Artificial Intelligence and Big Data (ICAIBD), At Chengdu, China.

2. J. CHAO W L, D. J J, L. J Z. Facial expression recognition based on improved local binary pattern and class-regularized locality preserving projection. Signal Processing, 2015, 117:1-10.

3. J. HU Liqiao, Q. Runhe. Face recognition based on adaptive weighted HOG. Computer Enigeering and Applications, 2017, 53(3): 164-168.

4. J. Yu yan JIANG, P. LI, Q. WANG. Labeled LDA model based on shared background topic. Acta Electronica Sinica, 2015, 2013, (9): 1794-1799.

5. J. WU Qi, W. Tang-hong, L. Zhan-li. Imporved face recognition algorithm based on Gabor feature and collaborative representation, Computer Engineering and Design, 2016, 37(10): 2769-2774.

6. A. Singh, S. Kumar Singh, S. Tiwari, Comparison of face Recognition Algorithms on Dummy Faces, The International Journal of Multimedia \& Its Applications (IJMA) Vol.4, No.4, August 2012.

7. X. Zhao, C. Wei, A Real-time Face Recognition System Based on the Improved LBPH Algorithm, 2017 IEEE 2nd International Conference on Signal and Image Processing.

8. V. Garg, K. Garg, Face Recognition Using Haar Cascade Classifier, Journal of Emerging Technologies and Innovative Research (JETIR), December 2016, Volume 3, Issue 12.

9. H. Zhang, Z. Qu Liping, Y. GangLi, A Face Recognition Method Based on LBP Feature for CNN, 2017 IEEE 2nd Advanced Information Technology, Electronic and Automation Control Conference (IAEAC).

10. T. Chen, Y. Wotao, S. Z. Xiang, D. Comaniciu, and T. S. Huang, "Total variation models for variable lighting face recognition" IEEE Transactions on Pattern Analysis and Machine Intelligence, 28(9): $1519\{1524,2006$

11. Z. and R. Chellappa "Robust face recognition using symmetric shape from-shading" Technical Report, Center for Automation Research, University of Maryland, 1999

12. Z. Xiang, H. Tan, W. Ye. The excellent properties of dense gird-based HOG features on face recognition compare to gabor and LBP, 2018 volume issue 99.

13. W. Chao, "Face Recognition",GICE,National Taiwan University.

14. L. Zhichao and Er. Meng Joo, "Face Recognition Under Varying Illumination", Nanyang Technilogical University, Singapore.

15. X. Sun, Q. Zhang and Z. Wang "Face Recognition Based on NMF and SVM", Wuhan University of Technology and Henan University of Technology, China, 2009.

16. Y. Hu, B. Liu "Face Recognition Based on PLS and HMM", Guizhou University, China, 2009.

17. A. Gubbi, Mohammad F. Azeem and N. Z H Nayakwadi "Face recognition using Local Ternary Pattern and Booth's Algorithm",3rd International Conference on Eco-Friendly Computing and Communication Systems, 2014.

18. Q. Li, C. Sun,Jingao Liu "Illumination Invariant Face Recognition Based on ULBP and SVM", JSNU, ECNU, China 5th International Conference on BioMedical Engineering and Informatics (BMEI 2012).

19. S. Y.Raut, D. A.Doshi "A Face Recognition System by Hidden Markov Model and Discriminating Set Approach", 2016

20. M. Rajapakse and L. Wyse "Face Recognition with Non-negative Matrix Factorization",Institute for Infocomm Research, Singapore.

21. Markov Model. Available at: https://in.mathworks.com/help/stats/hidden-markov-models-hmm.html [accessed on 12 march 2019]

22. K. Srinivasa Reddy, V.Vijaya Kumar, B. Eswara Reddy "Face Recognition Based on Texture Features using Local Ternary Patterns", Hyderabad, Hyderabad, A.P.,India.,2015

23. Proceedings of the International Conferences on Automatic Face and Gesture Recgonition, 1995-1998.

24. P.J. Phillips, P. Rauss, and S.Der, "FERET(Face Recognition Technology)

25. S. Lee and C. Lee, "Low complexity background subtraction based on spatial similarity", Eurasip Journal on Image and video processing, Springer, june, 2014.

26. E. Kermani and D. Asemani, "A robust adaptive algorithm of moving object detection for video surveillance", Eurasip Journal on Image and video processing, Springer, 2014. 
27. M. Piccardi, "Background Subtraction Techniques: a review", IEEE, International Conference on Systems, Man, Cybernatics, 2004.

28. K. Toyama, J. Krumm, B. Brumitt and B. Meyers, "Wallflower: Principles and Practice of Background Maintenance", IEEE Computer Society Press, Seventh International Conference on Computer Vision, Kerkyra, Greece, pp. 255-261, 1999.

29. L. Sharma, D. Yadav, "Histogram-based adaptive learning for background modelling: moving object detection in video surveillance", Internation Journal if Telemedicine and Clinical Practices, Inderscience, vol. 2, no.1, pp. 74-92,2017

30. L. Sharma, N. Lohan, "Performance analysis of moving object detection using BGS techniques in visual surveillance", Internation Journal of Spatiotemporal Data Science, Vol.1 No.1, pp.22-53,Jan.2019

31. C. Stauffer and W. E. L. Grimson, "Adaptive background mixture models for real-time tracking", IEEE Computer Society Conference on Computer Vision and Pattern Recognition, june, 1999.

32. J. Dou and J. Li, "Moving object detection based on improved VIBE and graph cut optimization", optic 124, pp. 6081-6088, Elsevier, 2013.

33. L. Lin, Y. Xu, X. Liang and J. Lai, "Complex background subtraction by pursuing dynamic spatio- temporal models", IEEE Trans. On image processing, vol. 23, no. 7, july 2014 .

\section{AUTHORS PROFILE}

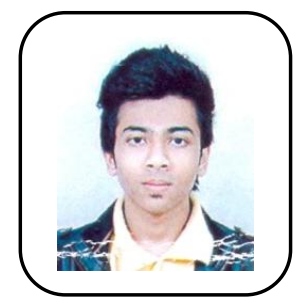

Akshit Anand is a BCA graduate in the field of information technology from Amity University. He had worked in National Small Industry Corporation, New Delhi. He has adequate knowledge of different programming languages. $\mathrm{He}$ also developed an Face recognition application in python using OpenCV libraries for recognizing in real time. His current research interests include AI - Machine Learning, Natural Language Processing and Internet of Things.

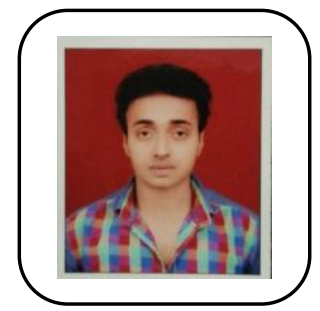

Vikrant Jha is a BCA graduate student in the field of information technology. He had worked in KR Network Cloud Technologies Pvt. Limited, Delhi. He also developed an Live HTML Editor Web application. And he has great knowledge in the field of Machine learning. His current research interests include AI - Machine learning and Internet of Things.

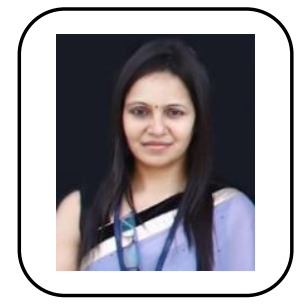

Dr. Lavanya Sharma is a Ph.D. graduate in the domain of Computer Vision at Uttarakhand Technical University (govt), India and an assistant professor, Amity Institute Information Technology at Amity University UP, Noida, India. She is an active nationally recognized researcher who produces dozens of papers in her field. She is an Editorial Member/Reviewer of various journal of repute and active program committee member of various IEEE and Springer conferences also She is an active nationally recognized computer vision educator who has produces dozens of papers. 